\title{
Sons de Korotkoff: desenvolvimento da pesquisa em esfigmomanometria na Escola de Enfermagem da USP
}

\author{
KOROTKOFF SOUNDS: DEVELOPMENT OF THE SPHYGMOMANOMETRY \\ RESEARCH AT THE NURSING SCHOOL OF THE USP
}

\author{
SONIDOS DE KOROTKOFF: DESARROLLO DE LA INVESTIGACIÓN \\ EN ESFIGMOMANOMETRIA EN LA ESCUELA DE ENFERMERÍA DE LA USP
}

Edna Apparecida Moura Arcuri', Thelma Leite de Araújo ${ }^{2}$, Eugênia Veludo Veiga ${ }^{3}$, Sonia Maria
Junqueira Vasconcellos de Oliveira $^{4}$, José Luiz Tatagiba Lamas ${ }^{5}$, Jair Lício Ferreira Santos ${ }^{6}$

\section{RESUMO}

Este artigo tem como objetivos reverenciar Sergei Nicolai Korotkoff por ocasião do centenário da descoberta do método auscultatório de medida da pressão arterial na Rússia, em 1905; relatar os fatos que culminaram no desenvolvimento da esfigmomanometria no Brasil; historiar a valiosa contribuição da Escola de Enfermagem da Universidade de São Paulo (EEUSP) no desenvolvimento da pesquisa, na área da medida da pressão e analisar o produto da linha de pesquisa "Influência da Largura do Manguito na Medida da Pressão Arterial", gerada na EEUSP a partir de 1974. O artigo relata a consolidação dos achados iniciais pelos estudos que permitiram a formação dos primeiros doutores na área, que sugere a confirmação de hipóteses em estudos longitudinais.

\section{DESCRITORES}

Auscultação/métodos. Pressão arterial.

Hipertensão/diagnóstico. Esfigmomanômetros/história.

\section{ABSTRACT}

This article pays homage to Sergei Nicolai Korotkoff, for the centenary of the auscultatory method discovery. It refers to his discover of blood pressure auscultatory method in Russia, in 1905; presents the evidence that resulted in the development of sphygmomanometry studies in Brasil; describes the important contribution of the Nursing School of the University of São Paulo to the development of blood pressure measurement research; and analyses the results of the research field of "Cuff width influence in blood pressure measurement", created at the Nursing School of The University of São Paulo, in 1974. The scientific results of doctoral studies are presented, identifying the research groups that are contributing to defying knowledge in this area. The article demonstrates the consolidation of the results related to the initial doctoral program studies developed in the area. Confirmation of hypothesis in longitudinal studies is emphasized.

\section{KEY WORDS}

Auscultation/methods.

Blood pressure.

Hipertension/diagnosis.

Sphygmomanometers/history.

\section{RESUMEN}

En este artículo se tuvo como objetivos reverenciar a Sergei Nicolai Korotkoff con motivo del centenario del descubrimiento del método de auscultación de medida de la presión arterial en Rusia, en 1905; relatar los hechos que culminaron en el desarrollo de la esfignomanometría en el Brasil; historiar la valiosa contribución de la Escuela de Enfermería de la Universidad de Sao Paulo (EEUSP) en el desarrollo de la investigación en el área de la medida de la presión y analizar el producto de la línea de investigación "Influencia del Ancho del brazalete en la Medida de la Presión Arterial", generada en la EEUSP a partir de 1974, evidenciando los estudios desarrollados en el curso de Postgrado (doctorado) en esta institución. El artículo relata la consolidación de los hallazgos iniciales de los estudios que permitieron la formación de los primeros doctores en el área, que sugiere la confirmación de hipótesis en estudios longitudinales.

\section{DESCRIPTORES}

Auscultación/métodos.

Presión sanguinea.

Hipertensión/diagnóstico.

Esfigmomanometros/historia.
1 Professora Titular da Universidade de Guarulhos. Professora Titular aposentada da Escola de Enfermagem da Universidade de São Paulo (EEUSP). earcuri@globo.com

2 Professora Adjunta do Departamento de Enfermagem da Universidade Federal do Ceará (UFC). thelma@ufc.br

3 Professora Livre Docente do Departamento de Enfermagem Geral e Especializada da Escola de Enfermagem de Ribeirão Preto (EERP) da USP. evveiga@eerp.usp.br 4 Professora do Departamento de Enfermagem MaternoInfantil e Psiquiátrica da EEUSP. soniaju@usp.br 5 Professor Doutor do Departamento de Enfermagem da Faculdade de Ciências Médicas da Universidade Estadual de Campinas (FCM-UNICAMP). zelamas@fcm.unicamp 6 Professor Titular do Departamento de Medicina Social da Faculdade de Medicina de Ribeirão Preto (FMRP) da USP. jalifesa@usp.br 


\section{INTRODUÇÃO}

Poucas comemorações foram planejadas para comemorar o centenário da descoberta dos Sons de Korotkoff em 1905, por Nicolai Sergei.Korotkoff ${ }^{(1)}$. A despeito do grande mérito do cientista russo, os eventos científicos de 2005 pouco lembraram o experimento que provocou perturbação no silencioso fluxo laminar sangüíneo na artéria braquial, gerando vórtices audíveis, o que possibilitou auscultar pela primeira vez, os sons que indicam os níveis das pressões sistólica e diastólica, durante a deflação do manguito. $\mathrm{O}$ grande esforço da indústria para introduzir no mercado esfigmomanômetros oscilométricos digitais, precisos ou não, e o desconhecimento pelos usuários da vulnerabilidade de muitos instrumentos facilmente descalibráveis, o que lhes diminui a precisão, talvez seja a principal razão do descaso à comemoração do estudo que foi fundamental ao desenvolvimento da cardiologia e nefrologia, além de todas as outras especialidades que necessitavam conhecer os níveis da pressão arterial, cuja medida constitui o procedimento básico de qualquer investigação de saúde.

Três anos antes da descoberta do método auscultatório foi identificada a primeira fonte de erro de medida da pressão, quando em 1901 Von Recklinghausen demonstrou que o uso do manguito do esfigmomanômetro introduzido por Riva Rocci em 1886, com 4,5 centímetros de largura, resultava em registros elevados de pressão e propôs o manguito com $12 \mathrm{~cm}^{(2)}$. Vale ressaltar que o cientista italiano aperfeiçoou os manômetros existentes em época de medidas palpatórias, adicionando um manguito de borracha inflável ${ }^{(3)}$, o que propiciou a realização do experimento de Korotkoff. A literatura do último século apresenta centenas de publicações sobre as fontes de erros que tornam a medida da pressão imprecisa, o que prejudica o diagnóstico e tratamento da hipertensão arterial. O século atual foi iniciado por discussões calorosas, que geraram polêmicas entre as sociedades internacionais, referentes ao desaparecimento dos manô-metros de mercúrio, por razões ecológicas, e aos novos parâmetros para definir os níveis de pressão que indicam hipertensão ou o estado de pré-hipertensão ${ }^{(4-6)}$. Cabe ressaltar, contudo, que as novas definições dos limites da pressão arterial normal ou elevada e as tentativas de enfraquecimento e mesmo desaparecimento do método auscultatório de medida da pressão, ocorrem sem que se tenha conquistado ou aplicado todo o conhecimento disponível para controlar todas as variáveis que contribuem para tornar imprecisa a medida da pressão arterial.

O ano de 2005 deveria ser rico em discussões que direcionassem estudos esfigmomanométricos realizados com bases científicas mais sólidas, no qual a análise da literatura do século XX fosse o tema principal, pois importantes erros continuam sendo cometidos até pelas sociedades mais expressivas nas áreas de hipertensão e cardiologia, como é o caso das recentes recomendações da American Heart Association. Desde 1951 essa entidade afirma que para não ocorrer hipo ou hiperestimação da pressão arterial, a largura do manguito do esfigmomanômetro deve corresponder a $40 \%$ da medida da circunferência braquial. Embora mantendo esta recomendação neste ano de 2005, por razões práticas a Associação Americana definiu manguitos com $12 \mathrm{~cm}$ e $16 \mathrm{~cm}$ de largura para circunferências braquiais de 22 a $26 \mathrm{~cm}$ e 27 a $52 \mathrm{~cm}$, respectivamente ${ }^{(7)}$. No entanto, quatro dimensões de comprimento são definidas para a largura de $16 \mathrm{~cm}$. Qual é a vantagem, do ponto de vista prático, de recomendar quatro tamanhos de comprimentos, sem aproveitar a oportunidade de investimento para oferecer mais opções referentes à largura? A análise da literatura é árdua, por falta de formação na área poucos percebem que nos estudos que concluíram que o erro decorrente do comprimento inadequado é mais importante do que da largura, essa última variável não foi devidamente controlada, além do que tal afirmação desconsidera totalmente os princípios que embasam o fenomenal estudo de Von Recklinghausen e o fantástico trabalho de alguns cientistas na década de $30^{(8-10)}$.

A decisão da associação norte-americana exclui completamente o diagnóstico precoce e tratamento no adulto magro e no de peso normal com circunferência braquial menor que 29 $\mathrm{cm}$, o que atinge principalmente um grande número de mulheres, impedindo-lhes suficiente tratamento farmacológico após diagnóstico tardio, quando a doença já está bem adiantada. A aplicação das recomendações da American Heart Association na prática médica resultará também em imprecisão no diagnóstico de muitas pes-soas obesas, que poderão ser falsamente diagnosticadas e receber desnecessariamente drogas anti-hipertensivas, devido o uso do manguito com $16 \mathrm{~cm}$ de largura, caso suas circunferências braquiais sejam maiores que $40 \mathrm{~cm}$, ou também deixarem de ser diagnosticados se a circunferência for entre 27 e $38 \mathrm{~cm}$.

Os autores desta publicação, doutores na área da esfigmomanometria, constituem um dos poucos grupos internacionais com sólida formação e experiência na área da medida da pressão. Todos utilizaram o método auscultatório em seus estudos de doutorado e demais pesquisas, e todos declaram total confiança no método, que muitas vezes são mal avaliados por bioengenheiros e pesquisadores clínicos, que o dissociam de outros princípios que fundamentam a medida da pressão, comumente desrespeitados. Outro aspecto preocupante é que não tendo formação específica em esfigmomanometria, muitos clínicos, pesquisadores e assessores de agências de fomento não possuem elementos para 
avaliar a dimensão do prejuízo causado pelas fontes de erros, sobretudo o método que fundamenta o funcionamento dos esfigmomanômetros e a inadequação do tamanho do manguito. Esses erros interferem na análise de muitas variáveis associadas à medida da pressão, sobretudo as referentes aos fatores de risco para moléstias cardiovasculares e protocolos para avaliação de drogas anti-hipertensivas.

Nesse ano de comemorações do centenário dos Sons de Korotkoff os autores refletem sobre a história da esfigmomanometria no Brasil, os resultados dos experimentos propiciados pelo do uso do método auscultatório, que até hoje fundamenta o trabalho cotidiano em seus laboratórios e suas relações com as atividades de pesquisa, ensino e assistência. Historiam os fatos que marcaram, há cerca de 30 anos, o início do desenvolvimento de, talvez, a mais antiga linha de pesquisa na área da esfigmomanometria e enfermagem no Brasil, na intenção de oferecer um tributo à Escola de Enfermagem da Universidade de São Paulo.

Determinantes Históricos: os estudos aqui relatados originaram-se em 1974, quando não se encontravam respostas para explicar os baixos valores das pressões sistólica e diastólica registrados em mulheres jovens, durante o ensino do procedimento de medida da pressão aos acadêmicos da Escola de Enfermagem da Universidade de São Paulo (EEUSP). Tentativas de discutir o problema com especialistas em propedêutica, cardiologia e nefrologia, resultaram muitas vezes no comentário a pressão é normal, as mulheres têm mania de dizer que a pressão é baixa. Na época era grande a venda de drogas vaso-constrictoras para aumentar a pressão (quase exclusivamente no sexo feminino), sobretudo nos dias quentes de verão de um país tropical.

Por que minha pressão é baixa? No curso de fisiologia aprendo que o normal é 120 por 80 ( $\mathrm{mmHg}$, sistólica e diastólica, respectivamente), como é no rato, perguntavam as alunas. De fato era comum encontrar registros de $90 \mathrm{mmHg}$ na determinação da pressão siatólica e 50mmHg na diastólica, nos braços delgados daquela população jovem, pois as aulas ocorriam no mês de fevereiro, em pleno verão. Continuando sem respostas para tais perguntas, comuns durante a década de setenta, e não havendo pessoas que estudassem essa questão específica naquela época pré-internet, o livro High Blood Pressure, de Sir George Pickering ${ }^{(1)}$, adquirido por indicação da saudosa Professora Wanda de Aguiar Horta, constituiu-se no marco inicial da análise da literatura sobre a medida da pressão, procedimento este mais realizado pelas enfermeiras e médicos no mundo. Ressalta-se que encontrar o referido livro no acervo da biblioteca da EEUSP foi motivo de grande satisfação, pois se tratava de um dos raros exemplares no Brasil.

Revisão da literatura: além do conteúdo específico sobre hipertensão, o livro High Blood Pressure fazia referências de importantes contribuições na área da medida da pressão. Com a ajuda da Biblioteca Regional de Medi- cina (BIREME) pode-se ter acesso à literatura pertinente, cuja aquisição dava início a um rico acervo de esfigmomanometria, o qual foi de fundamental importância na formação de mestres e doutores na área. Alguns anos após apesar de importantes estudos realizados na década de 30 indicarem que o manguito padrão poderia causar também hipoestimação da pressão, toda a literatura após os anos 40 voltava-se apenas para a hiperestimação em braços grossos. Referências ao fenômeno contrário, a hipoestimação decorrente do uso do manguito com $12 \mathrm{~cm}$ de largura em braços finos, ficaram restritas às evidências apontadas nos estudos já referidos, realizados em animais, crianças e adultos, não constituindo um problema que preocupasse os clínicos na segunda metade do século. Controvérsias decorrentes de diferentes achados em pesquisas esfigmomanométricas começaram a surgir a partir dos estudos de Ragan e Bordley e de Holland e Humerfelt, depois de 1940 ${ }^{(12-13)}$. Essas controvérsias foram suficientes para que Pickering refletisse sobre a tabela de correção de erros que propusera alguns anos antes, como referido em seu livro ${ }^{(11)}$.

Em 1951 a American Heart Association ${ }^{(14)}$ passou a incluir, em suas recomendações, a informação de que a largura do manguito deveria ser $20 \%$ maior do que o diâmetro do braço (cerca de $40 \%$ da circunferência braquial). Contudo, logo constatamos que tal referência nunca havia sido testada e a atenção era direcionada apenas para a hiperestimação da pressão nos obesos, desde as primeiras investigações clínicas $^{(15-16)}$, sobretudo nos dias atuais quando é grande o desafio norte-americano de combate à obesidade.

Após a compreensão da literatura questionou-se: Se para evitar hiper ou hipoestimação era indicado que o manguito deveria ter a largura específica para cada braço, o que demandava diversas larguras na prática assistencial poderia então o manguito de largura padrão estar subestimando a pressão das alunas e mulheres no campo assistencial? Por que os autores não utilizaram manguitos que atendiam a razão de 0,40 entre a largura e a circunferência braquial? Destaca-se que todas as investigações que tentaram observar a influência da largura ou comprimento do manguito na medida da pressão, os autores faziam uso apenas das larguras disponíveis no mercado, a largura padrão de $12 \mathrm{~cm}$, e poucas larguras maiores, destinadas aos braços mais grossos. A maioria dos estudos foram realizados em populações com alterações clínicas, sobretudo cardíacas, nefrológicas e metabólicas, e até em clínica para perda de peso $^{(17)}$, o que facilitava a composição da amostra, ou realizadas em modelos rígidos de laboratórios ${ }^{(18)}$, não incluindo, via de regra, população sadia jovem.

A busca de respostas às questões formuladas pelos alunos e a análise do conhecimento disponível na área, resultou no início de uma linha de pesquisa em esfigmomanometria na EEUSP, iniciada por um estudo desenvolvido em 100 crianças de 10 a 14 anos e 900 adultos atuantes na USP (alunos, docentes e funcionários), de 17 a 59 anos, 450 
mulheres e 450 homens ${ }^{(19)}$. Um dos referenciais teóricos importantes na adoção de um método que garantisse o controle de todas as variáveis referentes aos erros de medida, foi a instrução programada Correcting common errors in indirect blood pressure measurement ${ }^{(20)}$, utilizada durante muitos anos no ensino do Laboratório de Hipertensão da EEUSP. Outro estudo também localizado no acervo da EEUSP foi uma pesquisa inédita apresentada na convenção da American Nursing Association (ANA-1961) por Jane Wilcox, cuja tese de doutorado constituiu-se numa das primeiras investigações sobre os erros provocados pelo observador (pessoa que mede a pressão) $^{(21)}$. Mais uma vez a biblioteca da EEUSP dispunha do material mais abrangente sobre as fontes de erros de medida, o que a tornava o melhor acervo nacional na área por reunir no assunto a literatura de enfermagem e médica.

Os outros detalhes sobre o controle das fontes de erros de medida no método adotado, foram subsidiados pela realização de créditos em neurofisiologia no Instituto de Ciências Biomédicas-USP, sobretudo o controle do alerta, a partir das magistrais aulas do saudoso Professor César Timo Iaria, além dos cursos de hemodinâmica (Prof. Maurício Rocha e Silva) e regulação cardiovascular e choque (saudoso Prof. Pedro Guertzeinstein e prof. Oswaldo Ubríaco Lopes). Fazse necessário lembrar que a elaboração do protocolo foi anterior à publicação das recomendações da American Heart Association de 1980 e antes que o fenômeno White Coat Hypertension (alterações emocionais responsáveis pelo aumento da pressão diante do médico, conhecido em nosso meio como Fenômeno do Jaleco Branco) ${ }^{(22)}$, fosse conhecido pela comunidade científica e clínica (com raras exceções). As bases teóricas referidas fundamentaram os passos metodológicos da verificação da pressão arterial. Outro fato destacável é que naquele tempo ainda não existia em São Paulo cursos de Doutorado em Enfermagem, o que resultava na procura pelos mestres da EEUSP de outros locais para esse nível de formação, o que fez com que a pesquisa realizada por Arcuri fosse formalmente vinculada ao Doutorado em Ciências (Fisiologia, concentração na área cardiovascular), no Instituto de Ciências Biomédicas da USP.

Além da linha de pesquisa denominada inicialmente Influência da Largura do Manguito na Medida da Pressão Arterial ter nascido na EEUSP, qual foi o principal papel dessa instituição no desenvolvimento da esfigmomanometria no Brasil? Destaca-se aqui o desafio que representou na época construir um kit com vários manguitos, pois o objetivo principal da pesquisa era medir a pressão nos sujeitos da amostra utilizando manguitos cujas larguras atendessem as dimensões de seus braços (o comprimento era sempre o dobro da largura, atingindo $80 \%$ da circunferência braquial, conforme recomendado) e comparar os resultados com os obtidos com o manguito de largura padrão, já que a própria associação norte-americana não o havia testado. A procura de manguitos no mercado nacional e internacional nos levou a compreensão das dificuldades na obtenção de larguras não padronizadas, o que fragilizou os resultados de muitas investigações descritas na literatura internacional. No entanto, havia um estudo pioneiro na própria EEUSP, no qual haviam sido utilizados manguitos infantis de pequenas dimensões, relacionado à tese de doutorado da docente Dirce Maria Rodrigues Martins ${ }^{(23)}$. Foi então que constatamos que a Escola de Enfermagem da USP foi a primeira instituição a usar, internacionalmente, manguitos cujas larguras aproximavam-se da razão circunferência braquial/largura de manguito de 0,40 em cada braço, atendendo as necessidades individuais para se garantir uma medida precisa, evitando hipo ou hiperestimação no registro da pressão.

Os Kits com manguitos com largura variando a cada centímetro foram providenciados por funcionários da seção de patrimônio da EE. As bolsas de borracha eram confeccionadas na oficina de reparos Coreme, que logo deixou de existir após a morte de seu proprietário. A borracha utilizada na fabricação das bolsas era da melhor qualidade já conhecida pelos pesquisadores, pois após 1985 alguns kits feitos por outros fabricantes, adquiridos no mercado interno, não chegaram a resistir a um experimento. As braçadeiras de tecido também foram confeccionadas na própria escola, que tinha na época uma seção especializada em costura. Diante das dificuldades na aquisição de manguitos na década de 90, a boa qualidade do material da EEUSP permitiu que fosse utilizado por longo tempo em outros centros, como em projeto de doutorado na EERPUSP, voltado para o estudo da pressão arterial em escolares.

Resultados do estudo: a aplicação da recomendação da associação norte-americana referente à largura do manguito, num protocolo que representou uma avaliação do manguito padrão, demonstrou que a maioria da população estudada necessitava de manguitos menores que o padrão, tendo este causado preocupante hipoestimação nos registros de pressão. Os dados obtidos com o manguito correto revelaram que a pressão da mulher não é baixa, pois se trata de erro de avaliação causado pelo uso do manguito padrão nos braços jovens, assim como nos idosos magros. Os registros indiretos de pressão efetuados em 795 adultos normotensos resultaram na média mais próxima dos níveis basais intrarteriais sistólicos até então encontrados na literatura, 119,0 $\mathrm{mmHg}$, o que fez a autora denominar o manguito individual de manguito correto.

Resultados surpreendentes foram observados quando o manguito correto foi utilizado, registrando níveis de pressão em faixas hipertensivas, porém após um minuto o manguito padrão registrava níveis normais. Tais discrepâncias motivaram a realização de outros protocolos para validar o método ${ }^{(24)}$, com repetição do estudo em crianças ${ }^{(25)}$, gestantes $^{(26)}$, comparações com registros intrateriais ${ }^{(27)}$ e concomitantemente estudos que visavam identificar o conhecimento de enfermeiros sobre a medida da pressão arterial ${ }^{(28)}$. 
Esses estudos foram realizados por quatro docentes enfermeiros que escolheram a área para se desenvolver em nível de doutorado, dois da EEUSP, um da EERP-USP e um da UNICAMP (cuja seqüência na identificação dos autores deste trabalho obedece à seqüência e a cronologia do tempo de defesa de suas teses). A consonância cada vez maior entre os dados dessas investigações e os achados do estudo inicial foi adicionando consistência à linha de pesquisa, tornando-a cada vez mais sólida. Destaca-se também um estudo em nível de mestrado, realizado por outra docente da EEUSP, voltado para registros de pressão em função da largura do manguito no período pré-operatório, de recuperação e pós-anestésico ${ }^{(29)}$.

Paralelamente aos estudos que visavam à compreensão da influência da largura do manguito na medida da pressão, Pierin docente da EEUSP, desenvolveu o primeiro estudo no Brasil utilizando a Moni-toração Ambulatorial da Pressão Arterial (MAPA) ${ }^{(30)}$, investigação vinculada ao doutorado da EEUSP. Essa docente tornou-se referência nacional na área de esfigmomanometria no país, graças à facilidade com que ampliou os conhecimentos adquiridos no ensino e atividades assistenciais junto a Liga de Hipertensão do Hospital das Clínicas (HCUSP). O estudo realizado ${ }^{(30)}$ comparou dados registrados no domicílio com os obtidos em ambulatórios, na tentativa de identificar o Fenômeno do Jaleco Branco (White Coat Hypertension ), que é o aumento da pressão arterial, sobretudo na presença do médico, devido alterações no estado de alerta por razões emocionais. Sua linha de pesquisa nasceu do trabalho realizado na Liga de Hipertensão, razão porque entendemos que seu autodidatismo, extensa produção, anos de ensino voltado para assistência ao portador de hipertensão e a contribuição para o êxito das programações em datas comemorativas da área, merecem uma análise própria da pesquisadora.

O resultado mais preocupante no estudo realizado foi constatar, cada vez mais, que o manguito padrão retardava o diagnóstico da hipertensão, em pessoas cujas circunferências braquiais eram menores que $28 \mathrm{~cm}$. Não havendo outra investigação descrita na literatura a respeito da falta de diagnóstico pela inadequação do manguito padrão em braços não obesos, decidiu-se acompanhar alguns participantes do estudo, nos quais a ausência de diagnóstico precoce resultou em complicações como descolamento prematuro da placenta, infarto do miocárdio, pré-infarto, arritmias e sintomas relacionados à hipertensão, como cefaléia, cansaço, taquicardia. Vale destacar que desde o início, a tentativa de discussão dos achados no Brasil foi frustrada por falta de pessoas que conhecessem a literatura, mesmo entre líderes da área de hipertensão, apesar das tentativas feitas em 1985, durante o congresso anual das áreas de hipertensão, cardiologia e investigação clínica (sociedades integradas na época), o que resultou em estudos pós-doutorais nas universidades de Oxford e Milão em 1986
A escolha dos tradicionais centros de estudos para a realização do programa de pós-doutorado, iniciando pelo laboratório desenvolvido por Sir George Pickering, foi de fundamental importância ao início da discussão dos achados brasileiros, que foram discutidos em apresentações formais (lectures). Entretanto, a motivação para dar seqüência aos estudos longitudinais resultou da discussão dos achados com o Professor Rose, epidemiologista da Escola se Saúde Pública de Londres, o qual comentou sobre a grande dificuldade de tratamento do indivíduo magro hipertenso, sua falta de resposta à droga anti-hipertensiva e possíveis relações desses problemas com os dados do estudo. Este fato foi fundamental para que continuássemos acompanhando as pessoas que nos preocupavam ao retornar ao Brasil em 1987, época em que diversas investigações demonstravam o favorecimento do tratamento no obeso ${ }^{(31)} \mathrm{e}$ a maior mortalidade no magro hipertenso ${ }^{(32)}$.

Em 1989 foram levantadas hipóteses na tentativa de explicar a causa do insucesso do tratamento das pessoas hipertensas magras (Congresso Europeu de Hipertensão, Milão). Sugerimos que a hipoestimação provocada pelo manguito de largura padrão em braços finos, fazia com que as pessoas ficassem anos sem diagnóstico e quando tardiamente diagnosticadas, recebiam menor quantidade de drogas anti-hipertensivas que necessitavam. Tais hipóteses foram publicadas nos periódicos Arquivos Brasileiros de Cardiologia ${ }^{(33)}$ (Conferência, escrita a convite do eminente Professor Luiz Décourt), no Journal of Hypertension $^{(34)}$, ambos em 1989. Como essas hipóteses fundamentavam-se em apenas 11 pessoas acompanhadas, novos desafios resultaram em estudos longitudinais para confirmá-los em amostra mais representativa.

No Congresso da Sociedade Internacional de Hipertensão (ISH) em 2004 foram apresentados os resultados do estudo longitudinal de 20 anos de acompanhamento de 492 participantes, que formaram dois grupos: um que apresentou em 1983 níveis sistólicos e ou diastólicos elevados apenas com a utilização do manguito correto (grupo experimental, 237 pessoas) e outro com registros de pressão normais, com o manguito correto ou padrão (Grupo Controle, 255). O objetivo foi verificar freqüência de hipertensão diagnosticada com o manguito padrão, nos serviços médicos onde eram atendidos os sujeitos dos dois grupos. Os dados foram obtidos por meio de entrevistas, nas unidades da USP, em domicílios, ou via telefone ou internet, pois muitos docentes e funcionários já não atuavam na Universidade, devido à aposentadoria ou outras razões. A maior dificuldade foi entrevistar estudantes e docentes no Exterior, que foram localizados no Japão, Israel, Alemanha, Suíça, Estados Unidos, e no Brasil, no sertão de Pernambuco, Rio Branco, entre outros estados. Os resultados confirmaram nossas hipóteses, pois enquanto $57 \%$ das pessoas do grupo experimental tornaram-se hipertensas, no grupo controle esta ocorrência não ultrapassou $26 \%$. Achado mais significativo foi verifi- 
car que de 48 mortes, 37 encontravam-se no grupo experimental, dessas $30 \%$ por razões cardiovasculares, o que deve resultar em novas investigações ${ }^{(35)}$.

No Congresso da Sociedade Interamericana de Hipertensão em Cancun-2005, foram apresentados dados comprovando que quanto mais fino o braço, maior a ocorrência e a gravidade das complicações cardiovasculares detectadas. A freqüência de complicações foi inversamente proporcional ao tamanho de manguito, sendo que algumas pessoas extremamente magras, que apresentaram complicações da hipertensão, não foram devidamente diagnosticadas pelo manguito padrão até os dias atuais ${ }^{(36)}$. O diagnóstico tardio é geralmente feito durante crises hipertensivas, porém em pessoas muito magras é comum interpretar o quadro hipertensivo como questão de estresse, porque seus braços finos não permitem avaliação correta com o manguito padrão nos hospitais. Uma funcionária da Universidade, cujo braço era o mais fino da amostra (48 anos), procurou o hospital devido forte cefaléia, foi medicada com analgésicos e morreu dois dias depois por AVC, antes do tratamento adequado. Esta funcionária trabalhava em uma das bibliotecas da universidade e suas frequientes cefaléias sempre nos preocuparam, porém durante muitos anos ela foi considerada normotensa nas avaliações médicas e o diagnóstico de hipertensão foi retardado pelo manguito padrão, que também lhe impediu a quantidade de drogas antihipertensivas suficientes, mesmo após o diagnóstico. Infartos e outras complicações cardíacas, acidentes cardiovascular cerebral, pré-eclampsia, eclampsia e descolamento prematuro da placenta estiveram presentes em várias pessoas que foram acompanhadas ao longo de 20 anos.

Constatar tristes episódios que começaram a fazer parte das atividades cotidianas dos pesquisadores resultou em grande reflexão sobre o sistema de saúde, que hoje conta com sofisticada tecnologia para diagnosticar e tratar as complicações da hipertensão, não havendo determinação suficiente para enfrentar os desafios impostos ao diagnóstico preciso e precoce. Tal fato resulta em esforços e esperança de que um dia esses problemas possam ser observados e acreditados por outros investigadores, pois após um século apenas o diagnóstico impreciso do obeso é motivo de preocupação.

\section{REFERÊNCIAS}

1. Korotkoff NS. To the question of methods of determining blood pressure. Rep Imp Milit Med Acad. 1905;2:365-7.

2. Von Recklinghausen H. Ueber blutdruckmessun beim menschen. Arch Exp Pathol Pharmakol. 1901;46(1):78-132.

3. Riva-Rocci S. Un nuovo sfigmomanometro. Gazzeta Medica Torino. 1896; 47:981-1001.

\section{CONSIDERAÇÕES FINAIS}

Segundo o Conselho Internacional de Enfermeiras e a OMS, a medida da pressão arterial é o procedimento mais realizado pelas enfermeiras e médicos no mundo. A pesquisa em esfigmomanometria é árdua, não se distinguindo na literatura grupos persistentes em continuar seus estudos e lutar contra as decisões equivocadas das sociedades da área muitas vezes tomadas sem evidência científica, visando apenas não complicar a prática e proteger os obesos. Se é inviável ter diversos manguitos disponíveis, incentivos devem ser dados para que bioengenheiros pesquisem alternativas para a medida da pressão, já que após um século de esfigmomanometria, pessoas morrem por AVC sem a chance de diagnóstico e tratamento correto, além de outros tipos de complicações cardiovasculares.

O grupo de enfermeiros brasileiros especialistas na área vai aos poucos ampliando e solidificando conhecimentos. Os doutores formados desenvolveram novos núcleos em suas unidades de origem ou em outras regiões do país, o que vem resultando em considerável número de projetos em nível de iniciação científica e mestrado. Os autores não medem esforços para enfrentar os desafios impostos na tentativa de divulgar seus achados, esperando que um dia suas pesquisas possam ajudar na resolução dos problemas concernentes as relações da medida da pressão arterial e diagnóstico de hipertensão.

Nesta publicação procurou-se homenagear Korotkoff pelo centenário do método auscultatório, assim como oferecer um tributo à Escola da Enfermagem da Universidade de São Paulo, pelo importante apoio na formação dos autores deste relato, propiciando-lhes a oportunidade de desenvolvimento da linha de pesquisa aqui relatada. Em próximo artigo serão abordados aspectos da avaliação quantitativa e qualitativa na produção dos doutores formados, com base em indicadores de caracterização de conhecimento e principais questões respondidas pelos estudos efetuados.

4. Chobanian AV, Bakris GL, Black HR, Cushman WC, Green LA, Izzo JL, et al. The seventh report of the Joint National Committee on Prevention, Detection, Evaluation, and Treatment of High Blood Pressure. Hypertension. 2003;42(6):1206-52.

5. European Society of Hypertension-European Society of Cardiology Guidelines Committee 2003 European Society of Hypertension-European Society of Cardiology guidelines for the management of arterial hypertension. J Hypertens. 2003;21(6):1011-53. 
6. Sociedade Brasileira de Cardiologia. Sociedade Brasileira de Hipertensão. Sociedade Brasileira de Nefrologia. Diretrizes Brasileiras de Hipertensão Arterial, 4, 2002. São Paulo: BG Cultural; 2002.

7. Pickering TG, Hall JE, Apple LJ, Falkner BE, Graves J, Hill $\mathrm{MN}$, et al. Recommendations for blood pressure measurement in humans and experimental animals. Hypertension. 2005;45 (1):142-61.

8. Von Bonsdorff B. Zur methodik der blutdruk messung in acute hypertension. Acta Med Scand. 1933;51/52:1-197.

9. Bazett HC, Laplace LB. Studies on indirect measurement of blood pressure: I- Sources of error in the Riva Rocci Method. Am J Physiol. 1933;103(1):48-67.

10. Woodbury RA, Robinow M, Hamilton WF. Blood pressure studies on infants. Am J Physiol. 1938;122:472-9.

11. Pickering G. High blood pressure $2^{\text {nd }}$ ed. London: Churchill Livingtone; 1968. p.17-21.

12. Ragan C, Bordley III J the accuracy of clinical measurements of arterial blood pressure. Johns Hopkins Bull. 1941;69:504-28.

13. Holland WW, Humerfelt S. Measurements of blood pressure comparison of intra-arterial and cuff values. Br Med J. 1964; 2(5419):1241-3.

14. Bordley III J, Connor AR, Hamilton WF, Kerr WJ, Wigger CJ. Recommendations for human blood pressure determinations by sphygmomanometers. Circulation. 1951;4(4):503-9.

15. Berliner K, Fujiy H, Lee D, Yildiz M, Garnier B. Accuracy of blood pressure determinations: comparison of direct and indirect measurements. Cardiologia. 1960;37(1):118-28.

16. Ostchega Y, Dillon C, Carroll M, Prineas RJ, McDowell M. US demographic trends in mid-arm circumference and recommended blood pressure cuffs: 1982-2002. J Human Hypertens. 2005;19(11): 885-91.

17. Maxwell MH, Warks AV Schroth PC, Karan M, Dornfeld LP. Error in blood pressure measurement due to incorrect cuff size in obese patients. Lancet. 1982; 2(8288):33-5.

18. Alexander H, Cohen ML, Steinfeld L. Criteria in the choice of an occluding cuff for the indirect measurement of blood pressure. Med Biol Eng Comp. 1977;15(1):2-10.

19. Arcuri EAM. Estudo comparativo da medida da pressão arterial com o manguito de largura correta e com o manguito de largura padrão [tese]. São Paulo: Instituto de Ciências Biomédicas, Universidade de São Paulo; 1985.

20. Malcolm B, Glor B. Program med instruction: correcting common errors in blood pressure measurement. Am J Nurs. 1965;65(10):133-64.

21. Wilcox J. Observer factors in the measurement of blood pressure. Nurs Res. 1961;10:4-17.

22. Mancia G, Zanchetti A. Ambulatory blood pressure monitoring in hypertension. Clin Exp Hypertens. 1993;15(6):1099-108.
23. Martins DMR. Estudo da pressão arterial no primeiro ano de vida [dissertação]. São Paulo: Escola de Enfermagem, Universidade de São Paulo; 1978

24. Arcuri EAM. Medida da Pressão pelo método auscultatório: necessidade de utilização de manguito de largura adequada para detecção precoce da hipertensão [tese livre-docência]. São Paulo: Escola de Enfermagem, Universidade de São Paulo; 1985.

25. Veiga EV. Medida direta da pressão arterial em função da largura do manguito, em escolares de 6 a 10 anos de idade [tese]. Ribeirão Preto: Escola de Enfermagem de Ribeirão Preto, Universidade de São Paulo; 1995.

26. Oliveira SMV. Influência da largura do manguito na medida da pressão arterial no ciclo grávido-puerperal [tese]. SãoPaulo: Escola de Enfermagem, Universidade de São Paulo; 1997.

27. Lamas JLT. Medida da pressão arterial em locais alternativos: comparação de valores diretos e indiretos em função da largura do manguito [tese]. São Paulo: Escola de Enfermagem, Universidade de São Paulo; 1999.

28. Araújo TL. Medida indireta da pressão arterial: aspectos conceituais e caracterização do conhecimento do enfermeiro [tese]. São Paulo: Escola de Enfermagem, Universidade de São Paulo; 1994.

29. Peniche ACG. Medida da pressão arterial em função da largura do manguito em pacientes nas fases pré-operatória, recuperação e pós-anestésica [dissertação]. São Paulo: Escola de Enfermagem, Universidade de São Paulo; 2000.

30. Pierin AMG. Medidas da pressão arterial no ambulatório pelo cliente, enfermeira e médico comparadas a registros domiciliares [tese]. São Paulo: Escola de Enfermagem, Universidade de São Paulo; 1992.

31. Barret-Connor E, Khaw KT. Is hypertension more benign when associate with obesity? Circulation. 1985;72(1):53-60.

32. Goulbourt U, Holtzman E, Cohen-Mandelzwieg L, Neufeld $\mathrm{HN}$. Enhanced risk of coronary heart disease mortality in lean. Hypertension. 1987;10(1):22-8.

33. Arcuri EAM, Rocha e Silva M, Santos JLF. Manguito do esfigmomanômetro e diagnóstico de hipertensão. Arq Bras Cardiol. 1989;52(4):181-3.

34. Arcuri EAM, Rocha e Silva M, Santos JLF. Is early diagnosis of hypertension a function o cuff width? J Hypertens. Supll. 1989; 7(6):60-1.

35. Arcuri EAM, Martins E, Santos JLF. Correct versus standard cuff width: twenty years follow-up study. J Hypertens. 2004; 22(Supp11):137.

36. Arcuri EAM, Araújo TL, Veiga EV, Oliveira SMJV, Lamas JL, Santos JLF. The challenge of precise blood pressure measurement. In: Proceedings of the 16th Scientific Meeting of the Interamerican Society of Hypertension Cancun. Dallas: American Hearth Association; 2005. p. 185 